\title{
Quadrupedal coordination of bipedal gait: implications for movement disorders
}

\author{
Dietz, V
}

\begin{abstract}
During recent years, evidence has come up that bipedal locomotion is based on a quadrupedal limb coordination. A task-dependent neuronal coupling of upper and lower limbs allows one to involve the arms during gait but to uncouple this connection during voluntarily guided arm/hand movements. Hence, despite the evolution of a strong cortico-spinal control of hand/arm movements in humans, a quadrupedal limb coordination persists during locomotion. This has consequences for the limb coordination in movement disorders such as in Parkinson's disease (PD) and after stroke. In patients suffering $\mathrm{PD}$, the quadrupedal coordination of gait is basically preserved. The activation of upper limb muscles during locomotion is strong, similar as in age-matched healthy subjects although arm swing is reduced. This suggests a contribution of biomechanical constraints to immobility. In post-stroke subjects a close interactions between unaffected and affected sides with an impaired processing of afferent input takes place. An afferent volley applied to a leg nerve of the unaffected leg leads to a normal reflex activation of proximal arm muscles of both sides. In contrast, when the nerve of the affected leg was stimulated, neither on the affected nor in the unaffected arm muscles EMG responses appear. Muscle activation on the affected arm becomes normalized by influences of the unaffected side during locomotion. These observations have consequences for the rehabilitation of patients suffering movement disorders.
\end{abstract}

DOI: https://doi.org/10.1007/s00415-011-6063-4

Posted at the Zurich Open Repository and Archive, University of Zurich

ZORA URL: https://doi.org/10.5167/uzh-54238

Journal Article

Published Version

Originally published at:

Dietz, V (2011). Quadrupedal coordination of bipedal gait: implications for movement disorders. Journal of Neurology, 258(8):1406-1412.

DOI: https://doi.org/10.1007/s00415-011-6063-4 


\title{
Quadrupedal coordination of bipedal gait: implications for movement disorders
}

\author{
Volker Dietz
}

Received: 28 March 2011/ Accepted: 19 April 2011/Published online: 8 May 2011

(c) Springer-Verlag 2011

\begin{abstract}
During recent years, evidence has come up that bipedal locomotion is based on a quadrupedal limb coordination. A task-dependent neuronal coupling of upper and lower limbs allows one to involve the arms during gait but to uncouple this connection during voluntarily guided arm/ hand movements. Hence, despite the evolution of a strong cortico-spinal control of hand/arm movements in humans, a quadrupedal limb coordination persists during locomotion. This has consequences for the limb coordination in movement disorders such as in Parkinson's disease (PD) and after stroke. In patients suffering PD, the quadrupedal coordination of gait is basically preserved. The activation of upper limb muscles during locomotion is strong, similar as in age-matched healthy subjects although arm swing is reduced. This suggests a contribution of biomechanical constraints to immobility. In post-stroke subjects a close interactions between unaffected and affected sides with an impaired processing of afferent input takes place. An afferent volley applied to a leg nerve of the unaffected leg leads to a normal reflex activation of proximal arm muscles of both sides. In contrast, when the nerve of the affected leg was stimulated, neither on the affected nor in the unaffected arm muscles EMG responses appear. Muscle activation on the affected arm becomes normalized by influences of the unaffected side during locomotion. These observations have consequences for the rehabilitation of patients suffering movement disorders.
\end{abstract}

\footnotetext{
V. Dietz $(\bowtie)$

Spinal Cord Injury Centre, Balgrist University Hospital,

Forchstr. 340, Ch-8008, Zürich, Switzerland

e-mail: vdietz@paralab.balgrist.ch
}

Keywords Bipedal gait · Locomotion · Quadrupedal coordination · Movement disorders · Parkinson's disease $\cdot$ Stroke

\section{Introduction}

The coordination of forelimb and hindlimb rhythmic activities is a main characteristic feature of quadrupedal locomotion [1]. Specialized neural circuits located in the caudal spinal cord [the so-called central pattern generator (CPG) for locomotion] organize hindlimb locomotor activity, whereas specialized circuits in the rostral spinal cord control forelimb movements [2, 3]. The coordination of both circuits is mediated by propriospinal neurons with long axons, which couple the cervical and lumbar enlargements of the spinal cord $[4,5]$.

In many respects, bipedal and quadrupedal locomotion share common spinal neuronal control mechanisms. As in quadrupeds, long projecting propriospinal neurons couple the cervical and lumbar enlargements in humans [6]. Furthermore, the coordination of limb movements during walking is similar in human infants [7, 8], adults [9, 10], and quadrupeds $[3,11]$.

Nevertheless, there are also distinct differences because the upper limb in primates has become specialized to perform skilled hand movements. The evolution of upright stance and gait, in association with a differentiation of hand movements, represents a basic requirement for human cultural development [12]. This review deals with the question in how far the coordination of gait is still based on a quadrupedal limb coordination and whether this control is impaired in patients suffering Parkinson's disease (PD) and stroke. 


\section{Neural organization of human locomotion}

This part of the review deals with the question of whether there is a task-dependent switch from a strong direct (i.e. monosynaptic) cortical-motoneuronal control of upper limb muscles during reaching [13] and skilled hand and finger movements [14], to a more indirect control by cervical propriospinal circuits during locomotion of healthy subjects. This indirect control appears to occur in quadrupeds such as the cat [15].

Recent research indicates that interlimb coordination during human locomotion is organized in a similar way to that in the cat (for review cf. [16]). Only during locomotion but not during sitting or standing, an unilateral tibial nerve stimulation is followed by reflex EMG responses in the proximal muscles of both arms $[17,18]$. The observations indicate that the quadrupedal coupling of the limbs during locomotion, i.e. the corticospinal excitation of upper limb motoneurons, is mediated by long propriospinal neurons in the cervical spinal cord $[17,19]$ or the brainstem.

Observations made in the rat indicate that the mesencephalic locomotor region might be involved in such a coupling. In vertebrates, a unilateral activation of the mesencephalic region produces symmetrical bilateral locomotion [20]. Correspondingly, in our approach to study the quadrupedal limb coordination [17] a unilateral afferent volley from the leg applied during locomotion might be translated by the mesencephalic locomotor region into EMG responses in arm muscles of both sides. Consequently, at present we assume that the reflexes described here are in fact mediated by the brainstem. Nevertheless, a contribution by other supraspinal centres can hardly be excluded. This coupling allows a task-dependent neuronal linkage of cervical and thoraco-lumbar propriospinal circuits controlling leg and arm movements during human locomotor activities.

A flexible coupling of thoraco-lumbar and cervical centres allows humans to use the upper limbs for manipulative and skilled movements or, alternatively, for locomotor tasks [16]. This implies a functional, task-dependent gating of neuronal pathways between the neuronal circuits controlling lower and upper limb muscles during walking, reflected in the arm swing as a residual function of quadrupedal locomotion (Fig. 1). This assumption is based mainly on indirect evidence. First, interlimb coordination and reflex modulation are similarly organized during locomotion in humans and cats [16]. Second, task-dependent coupling of upper- and lower-limb muscles occurs during walking, but not during skilled hand movements or during standing [18]. Third, in line with such a flexible coupling of cervical and lumbar interneuronal circuits, transmission through the indirect cortical-motoneuronal system seems to be inhibited during a precision hand task

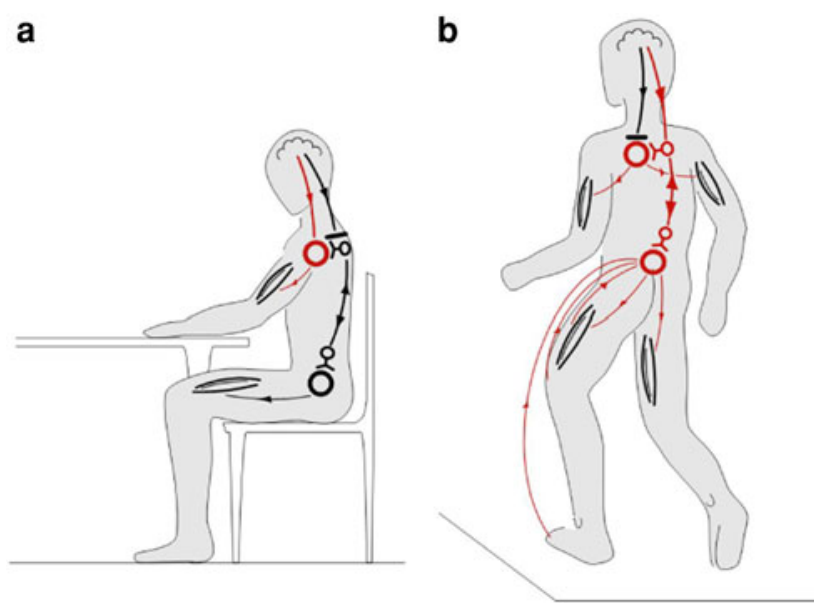

Fig. 1 Movement control during different motor tasks. According to recent research, neuronal control of arm movement is task-dependent. a During skilled hand movements a strong direct cortico-motoneuronal excitation is predominant (red lines) and the cervical propriospinal neuronal system is inhibited. b During locomotion, it is assumed that the brain command is predominantly mediated by interneurons. Cervical and thoraco-lumbar propriospinal systems become coupled and coordinate arm and leg movements (red lines). (From [16])

[18]. Fourth, during rhythmic movements of a foot, H-reflex modulation also involves upper limbs [21]. Finally, during locomotor activities such as swimming or crawling, there is a fixed coupling between upper and lower limb movements [22].

The demonstration of an interaction between cervical and thoraco-lumbar neuronal circuits in humans has relevance for the rehabilitation of patients suffering a damage within the central nervous system. For example, involvement of arm movements in the training, corresponding to experiments in spinalized cats [23], might have a positive effect on the locomotor capacity in these patients. In addition, the persistence of a quadrupedal movement control has consequences for the applicability of animal research to human patients with movement disorders and their underlying pathophysiology.

\section{Anticipatory spinal neuronal activity}

Recent studies on obstacle stepping demonstrate an anticipatory quadrupedal limb coordination with an involvement of proximal arm muscles in the acquisition and performance of a precision locomotor task [17]. This is presumably achieved by an up-regulated activity of coupled cervico-thoracal interneuronal circuits.

The unilateral non-noxious leg nerve stimulation during mid-stance allows us to study the excitability of spinal neuronal circuits. During obstacle stepping, reflex responses to unilateral tibial nerve stimulation in proximal arm muscles of both sides are enhanced in all arm and leg 
muscles prior to obstacle stepping compared with normal steps despite a low amplitude background EMG activity during mid-stance [17]. Following this reflex activity, the activation of proximal arm muscles was stronger during swing over the obstacle compared to normal swing. Thus, this reflex activity anticipates the following arm muscle activation [17]. Therefore, this reflex behaviour suggests an up-regulation of spinal interneuronal circuits that subserve such a precision locomotor task by coordinating arm and leg movements to keep the body balanced. Correspondingly, an up-regulation of reflexes is lacking when the body was stabilized by a partial unloading. In such a case, upper limb movements are less required for balancing the body during the performance of this task. The assumption that the enhanced reflex activity in arm and leg muscles prepares for the performance of an obstacle step is further supported by the observation that the muscle reflex response is not enhanced when subjects were informed that no obstacle approached (despite a warning signal) but continue normal walking [24].

It is assumed that the enhanced and modulated reflex responses prior to the obstacle step reflect an anticipatory action of spinal interneural circuits to prepare for the execution of the precision locomotor task. They might fulfil a similar function as the 'brain readiness potentials' preceding voluntary hand/arm movements $[25,26]$.

\section{Quadrupedal limb coordination in Parkinson's disease}

There are only a few studies focusing on the coupling of upper and lower limbs [27, 28] or the interleg coordination [29] during locomotion in subjects with PD. In this patient group, a defective coordination of upper and lower limbs [28, 30], in combination with reduced arm swing [27] during locomotion and abnormal postural reactions to voluntary movements [31], was suggested to contribute to the impaired performance of the locomotor tasks [32]. The disturbed interlimb coordination becomes improved by L-DOPA application and subthalamic nucleus stimulation [27]. Other known mechanisms contributing to the locomotor disorder in elderly people and PD subjects include an insufficient activation of leg extensor muscles [33] and a poor adaptation to environmental influences by a defective proprioceptive feedback [34].

Recently, the spinal interneuronal function underlying the quadrupedal limb coordination during gait and the performance in a precision, i.e. obstacle avoidance locomotor task was studied in PD subjects [35]. For this, the quadrupedal distribution of EMG responses to unilateral tibial nerve stimulation was analysed during locomotion prior to normal and obstacle swing, similar as done in healthy subjects [18]. Such a stimulation is known to evoke spinal reflexes in humans, most probably corresponding to cutaneous reflexes [36]. Up to now, only a few studies have investigated the behavior of cutaneous reflexes in PD subjects [37].

In such a condition, spinal reflex responses, evoked by tibial nerve stimulation during mid-stance, are present in all arm and leg muscles investigated in PD subjects [38]. They are larger before execution of obstacle avoidance movements compared with normal steps in the group of patients suffering PD and the group of age-matched healthy subjects. In both subject groups, i.e. PD and control subjects, spinal neuronal activity reflected in the spinal reflexes amplitudes is enhanced prior to obstacle step compared to normal swing, similar as in young healthy subjects [17]. Thus this mechanism appears to be basically preserved in PD subjects. Nevertheless, the anticipatory spinal reflex responses in the arm muscles prior to normal and obstacle steps are larger in Parkinson's disease compared with age-matched subjects.

As expected, the performance of obstacle stepping is slightly worse in PD than in control subjects. In both subject groups the arm and leg muscle activation is stronger during obstacle stepping compared with normal swing. These observations indicate that quadrupedal limb coordination is basically preserved in PD subjects [35]. The observations made are consistent with the proposal that in PD subjects an enhanced anticipatory spinal neuronal activity (reflected in the spinal reflex responses) in the arm muscles is required to achieve an appropriate muscle activation for the automatic control of body equilibrium during the performance of the task.

In line with an earlier report [32] subjects with moderate PD perform obstacle stepping almost as well as agematched healthy subjects, with the exception of a slightly higher foot clearance during obstacle steps and less adaptation. Compared to young subjects [17, 24], elderly healthy subjects also show a poorer performance. This fits with the observation that also elderly people have an increased risk of falls [33, 39].

Exploring the quadrupedal organization of bipedal gait in PD subjects no relevant arm movements are detected. However, proximal arm muscle activation is stronger (especially in the functionally relevant contralateral BB) [40] during swing over the obstacle compared to normal steps but does not differ between PD and elderly control subjects. Therefore, the contribution of upper limb muscle activation to the performance of the precision locomotor task is similar in both subject groups, despite the slightly worse performance of the task by PD subjects. Compared to young healthy subjects [17], the increase of arm muscle EMG during obstacle compared to normal swing is small in PD and control subjects. This attenuated modulation of arm muscle activity by the obstacle task (clinically probably 
reflected in a reduced arm swing) might contribute to the worse performance compared to the young healthy subjects [17].

Thus, PD subjects use a quadrupedal limb coordination. The enhanced anticipatory spinal neuronal activity might be required to achieve an appropriate arm muscle activation during task performance or might be directed to automatically compensate for the inherently reduced arm swing in PD subjects.

These observations indicate that also the slightly impaired quadrupedal neuronal coordination might contribute to the locomotor disorder in PD. Nevertheless, the results do not allow to speculate about their possible contribution to phenomena of $\mathrm{PD}$, such as gait freezing. According to the observations made, the goal to treat the gait disorder in PD might include strengthening the quadrupedal coordination of arm/leg muscle activation during the execution of specific locomotor tasks.

\section{Quadrupedal limb coordination in stroke subjects}

Arm movements are reduced on the paretic side of stroke subjects, although arm swing remains synchronized with stride frequency [41]. In addition, post-stroke subjects are able to adapt interlimb coordination of the legs to walk at different speeds on a split-belt treadmill [42]. Nevertheless, an abnormal coupling of upper and lower limb muscles was described in subjects following stroke [43-46] or cervical spinal cord lesions [19]. The disturbed inter- and intralimb coupling is assumed to contribute to falls in post-stroke subjects [47-52].

The use of reflex testing to investigate quadrupedal coupling of limb movements by spinal neuronal circuits during locomotion offers an approach to get more insight into several aspects of the movement disorder in stroke subjects. First, recording bilateral arm muscle reflex responses to unilateral tibial nerve stimulation prior to normal and obstacle swing allows the study of the taskmodulated processing of afferent input from the unaffected and affected legs and to probe the excitability of spinal neuronal circuits. In healthy subjects, an enhanced anticipatory spinal neuronal activity, mediating quadrupedal limb coordination prior to obstacle steps, takes place [17]. Second, bilateral arm muscle activation during normal and obstacle swing allows the study of automatic efferent control of arm movements. In healthy subjects, this arm muscle activation follows the preceding pattern of reflex activity and its course during repetitive steps [17].

In post-stroke subjects, the EMG responses in proximal arm muscles are stronger on both sides when the tibial nerve of the unaffected leg is stimulated during mid-stance compared with stimulation of the affected leg [53]. This difference is more pronounced when stimuli are applied prior to swing over an obstacle than prior to normal swing [53]. This indicates an impaired processing of afferent input from the affected leg resulting in attenuated and little task-modulated reflex responses in the arm muscles on both sides (Fig. 2). These observations suggest that a disrupted cortico-spinal control represents an important factor for the impaired processing of the afferent volley during locomotion of stroke subjects [54].

The mutual reflex interactions between the unaffected and affected sides of stroke subjects might explain the following observations. First, while the depression of presynaptic Ia inhibition is removed during the step cycle in patients with spinal injury, it is almost normal on the affected side in cerebral lesions [55]. Second, in a static condition, an abnormal stretch reflex activity is present not only on the affected but also on the unaffected side of stroke subjects [56].

In contrast to the reflex behaviour, activation of arm muscles are stronger during swing over an obstacle than during normal swing, with no difference in EMG amplitudes between the unaffected and affected sides. Therefore, it can be concluded that the deficits of the affected arm are compensated for by influences from the unaffected side. The observation that increased arm muscle activation is preserved in stroke subjects during obstacle steps on both sides, is at odds with the reflex behavior observed when the nerve of the affected leg is stimulated. Obviously, in poststroke subjects, the defective processing of afferent input from the affected leg can be compensated for by a dominance of the neuronal function arising from the unaffected leg. It remains unclear when this behavior emerges after a stroke.

Compared to the impaired processing of afferent input from the affected leg, the efferent part of the reflex pathway to the muscles of the affected arm seems to be only slightly impaired, i.e. the arm and leg muscle EMG responses are only slightly smaller on the affected side compared to the unaffected side when the leg nerve of the unaffected side is stimulated.

This discrepancy to the clinical impression might be explained by the fact that the assessment of the neurological deficit in stroke subjects (by the FIM score) includes both parts of sensori-motor dysfunction-the efferent deficit and the impaired afferent processing. In addition, the observations made concern the automatic movement control of locomotion which differs basically from voluntarily performed movements. These observations indicate strong mutual influences between unaffected and affected sides during locomotion of post-stroke subjects, which might be used to optimize rehabilitation approaches. 
A
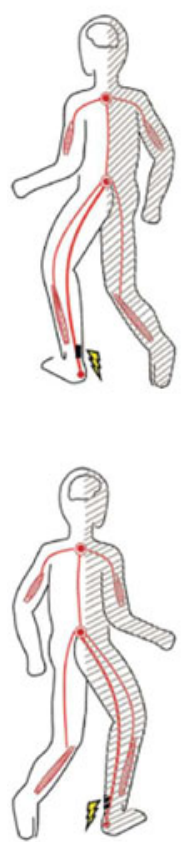

Normal Steps

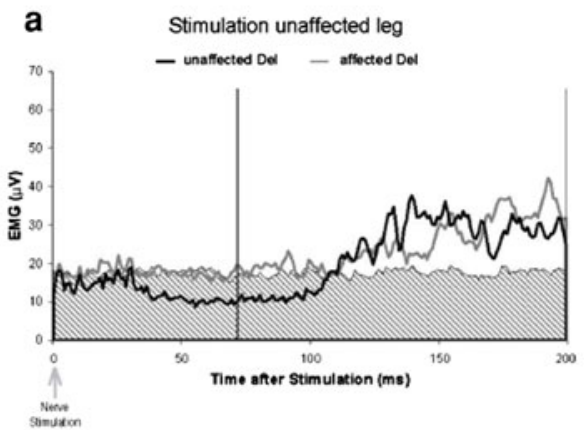

b Stimulation affected leg

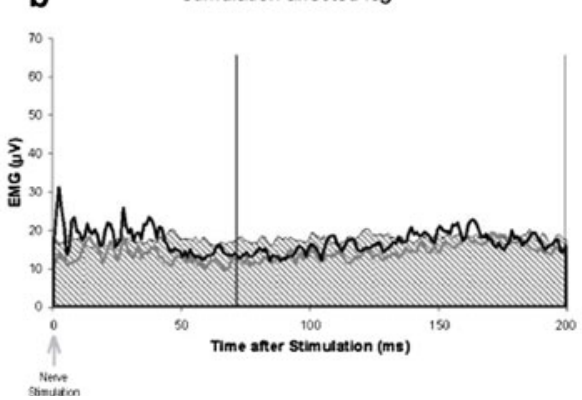

Fig. 2 Reflex responses in the deltoid muscles to tibial nerve stimulation. Grand means of the rectified reflex EMG responses in the unaffected (black lines) and the affected (grey lines) deltoid muscles to tibial nerve stimulation at the unaffected $(a)$ and affected (b) leg prior to normal (A) and obstacle (B) swing. In addition, the background EMG activity of non-stimulated steps of the unaffected

Despite a strong bilateral arm muscle activation observed during swing over an obstacle, arm swing is reduced on the paretic side of stroke subjects. A similar observation was made in PD subjects, who also show normal arm muscle activation, although arm swing was reduced compared to healthy subjects [35]. The assumption of biomechanical restraints might therefore also apply to the spastic paretic arm of stroke subjects [57].

As a functional consequence, a defective sensori-motor integration, which is assumed to be responsible for the disturbed inter- and intralimb coupling [47, 58], could essentially be due to the impaired processing of afferent input described here. The novel aspect of this research is that the reflex effects evoked by a non-noxious afferent volley concerns both the affected and unaffected arm of stroke subjects. The therapeutic consequence of the findings might have an influence on the recovery of gait and the effect of functional training in stroke subjects [59-61]. The combination of walking at maximum speed and having body weight support leads to marked speed-related improvements of locomotor ability, especially in low-functioning stroke subjects [62-64]. These effects should mainly be achieved due to sensori-motor interactions of the unaffected and affected sides during functional training. According to the actual

B

Obstacle Steps
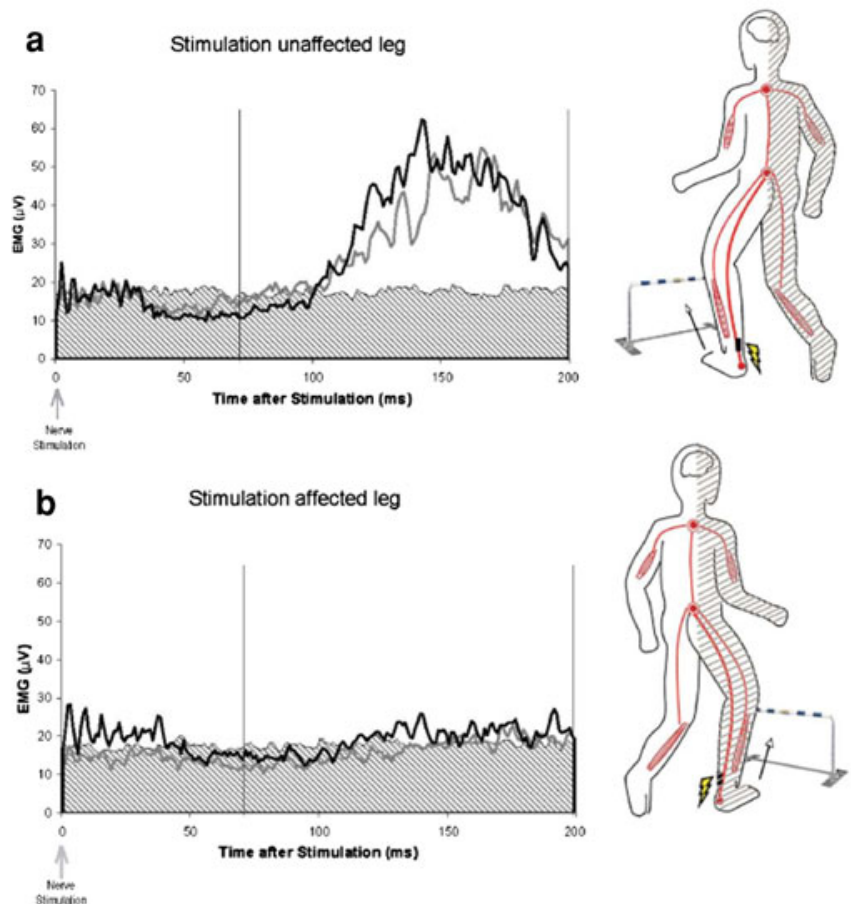

(upper graphs) and affected (lower graphs) sides are displayed (hatched areas). The reflex was randomly evoked by unilateral tibial nerve stimulation around mid-stance. The stimulus train (artifact) starts at $0 \mathrm{~ms}$ (vertical arrow) and lasts up to $40 \mathrm{~ms}$. The schematic drawings indicate the side of nerve stimulation (affected side hatched area) and the walking conditions [53]

findings described here, the effects of this training might be enhanced by providing additional afferent input from the unaffected side, especially in phases of the step cycle where balance reactions from the arms are required.

Conflict of interest None.

\section{References}

1. Gans C et al (1997) Vertebrate locomotion. In: Handbook of Physiology, Section 13: Comparative Physiology. Oxford University Press, England, pp 55-213

2. Duysens J et al (1998) Neural control of locomotion: the central pattern generator from cats to humans. Gait Posture 7:131-141

3. Grillner S (1981) Control of locomotion in bipeds, tetrapods and fish. In: Brookhart JMaMVB (ed) Handbook of physiology. The nervous system. Motor control. American Physilogy Society, New York, pp 1179-1236

4. Cazalets JR et al (2000) Coupling between lumbar and sacral motor networks in the neonatal rat spinal cord. Eur J Neurosci 12:2993-3002

5. Miller S et al (1975) Coordination of movements of the kindlimbs and forelimbs in different forms of locomotion in normal and decerebrate cats. Brain Res 91:217-237

6. Nathan PW et al (1996) Vestibulospinal, reticulospinal and descending propriospinal nerve fibres in man. Brain $119(\mathrm{Pt}$ 6):1809-1833 
7. Pang MY et al (2000) The initiation of the swing phase in human infant stepping: importance of hip position and leg loading. J Physiol 528 (Pt 2):389-404

8. Yang JF et al (1998) Transient disturbances to one limb produce coordinated, bilateral responses during infant stepping. J Neurophysiol 79:2329-2337

9. Dietz V (1992) Human neuronal control of automatic functional movements: interaction between central programs and afferent input. Physiol Rev 72:33-69

10. Dietz V (1997) Neurophysiology of gait disorders: present and future applications. Electroencephalogr Clin Neurophysiol 103:333-355

11. Grillner S (1986) Interaction between sensory signals and the central networks controlling locomotion in lamprey, dog fish and cat. In: Grillner $\mathrm{S}$ et al (eds) Wenner-Gren international symposium series. Neurobiology of vertebrate locomotion. Macmillan, England, pp 505-512

12. Herder JG (1785) Ideen zur Philosophie der Geschichte der Menschheit. Hartknoch, Leipzig

13. McKiernan BJ et al (1998) Corticomotoneuronal postspike effects in shoulder, elbow, wrist, digit, and intrinsic hand muscles during a reach and prehension task. J Neurophysiol 80:1961-1980

14. Porter R et al (1993) Corticospinal function and voluntary movement. Clarendon, Oxford

15. Alstermark B et al (1992) The C3-C4 propriospinal system: target-reaching and food-taking. In: Lea Jami (ed) Muscle afferents ans spinal control of movement. Pergamon, Oxford, pp 327-354

16. Dietz V (2002) Do human bipeds use quadrupedal coordination? Trends Neurosci 25:462-467

17. Michel J et al (2008) Obstacle stepping involves spinal anticipatory activity associated with quadrupedal limb coordination. Eur J Neurosci 27:1867-1875

18. Dietz V et al (2001) Neuronal coordination of arm and leg movements during human locomotion. Eur $\mathrm{J}$ Neurosci 14:1906-1914

19. Calancie B et al (1996) Central nervous system plasticity after spinal cord injury in man: interlimb reflexes and the influence of cutaneous stimulation. Electroencephalogr Clin Neurophysiol 101:304-315

20. Brocard F et al (2010) The transformation of a unilateral locomotor command into a symmetrical bilateral activation in the brainstem. J Neurosci 30:523-533

21. Baldissera $\mathrm{F}$ et al (1998) Cyclic modulation of the H-reflex in a wrist flexor during rhythmic flexion-extension movements of the ipsilateral foot. Exp Brain Res 118:427-430

22. Wannier $\mathrm{T}$ et al (2001) Arm to leg coordination in humans during walking, creeping and swimming activities. Exp Brain Res 141:375-379

23. De Leon RD et al (1999) Retention of hindlimb stepping ability in adult spinal cats after the cessation of step training. J Neurophysiol 81:85-94

24. Michel J et al (2007) Facilitation of spinal reflexes assists performing but not learning an obstacle-avoidance locomotor task. Eur J Neurosci 26:1299-1306

25. Kornhuber $\mathrm{HH}$ et al (1965) Changes in the brain potential in voluntary movements and passive movements in man: readiness potential and reafferent potentials. Pflugers Arch Gesamte Physiol Menschen Tiere 284:1-17

26. Shibasaki H et al (2006) What is the Bereitschaftspotential? Clin Neurophysiol 117:2341-2356

27. Carpinella I et al (2007) Effect of L-dopa and subthalamic nucleus stimulation on arm and leg swing during gait in Parkinson's Disease. Conf Proc IEEE Eng Med Biol Soc 2007:6665-6668
28. Winogrodzka A et al (2005) Rigidity and bradykinesia reduce interlimb coordination in Parkinsonian gait. Arch Phys Med Rehabil 86:183-189

29. Plotnik $M$ et al (2007) A new measure for quantifying the bilateral coordination of human gait: effects of aging and Parkinson's disease. Exp Brain Res 181:561-570

30. Swinnen SP et al (1997) Interlimb coordination deficits in patients with Parkinson's disease during the production of twojoint oscillations in the sagittal plane. Mov Disord 12:958-968

31. Rogers MW et al (1987) Postural adjustments preceding rapid arm movements in parkinsonian subjects. Neurosci Lett $75: 246-251$

32. van Hedel HJ et al (2006) Learning a high-precision locomotor task in patients with Parkinson's disease. Mov Disord 21:406-411

33. Dietz V et al (1998) Influence of body load on the gait pattern in Parkinson's disease. Mov Disord 13:255-261

34. Rogers MW (1996) Disorders of posture, balance, and gait in Parkinson's disease. Clin Geriatr Med 12:825-845

35. Dietz V et al (2008) Locomotion in Parkinson's disease: neuronal coupling of upper and lower limbs. Brain 131:3421-3431

36. Yang JF et al (1990) Phase-dependent reflex reversal in human leg muscles during walking. J Neurophysiol 63:1109-1117

37. Sandrini $G$ et al (2005) The lower limb flexion reflex in humans. Prog Neurobiol 77:353-395

38. Dietz V et al (2009) Human bipeds use quadrupedal coordination during locomotion. Ann N Y Acad Sci 1164:97-103

39. Nieuwenhuijzen PH et al (2006) Startle responses in Parkinson patients during human gait. Exp Brain Res 171:215-224

40. Grin L et al (2007) The effect of voluntary arm abduction on balance recovery following multidirectional stance perturbations. Exp Brain Res 178:62-78

41. Ford MP et al (2007) Phase manipulation and walking in stroke. J Neurol Phys Ther 31:85-91

42. Reisman DS et al (2007) Locomotor adaptation on a split-belt treadmill can improve walking symmetry post-stroke. Brain 130:1861-1872

43. Barzi Y et al (2008) Rhythmic arm cycling suppresses hyperactive soleus H-reflex amplitude after stroke. Clin Neurophysiol 119:1443-1452

44. Debaere F et al (2001) Coordination of upper and lower limb segments: deficits on the ipsilesional side after unilateral stroke. Exp Brain Res 141:519-529

45. Kline TL et al (2007) Exaggerated interlimb neural coupling following stroke. Brain 130:159-169

46. Stephenson JL et al (2010) The effect of arm movements on the lower limb during gait after a stroke. Gait Posture 31:109-115

47. Finley JM et al (2008) Stretch reflex coupling between the hip and knee: implications for impaired gait following stroke. Exp Brain Res 188:529-540

48. Divani AA et al (2009) Risk factors associated with injury attributable to falling among elderly population with history of stroke. Stroke 40:3286-3292

49. Lamontagne A et al (2009) Gaze and postural reorientation in the control of locomotor steering after stroke. Neurorehabil Neural Repair 23:256-266

50. Lamontagne A et al (2007) Physiological evaluation of gait disturbances post stroke. Clin Neurophysiol 118:717-729

51. Marigold DS et al (2006) Altered timing of postural reflexes contributes to falling in persons with chronic stroke. Exp Brain Res 171:459-468

52. Marigold DS et al (2004) Modulation of ankle muscle postural reflexes in stroke: influence of weight-bearing load. Clin Neurophysiol 115:2789-2797

53. Kloter E et al (2011) Locomotion in stroke subjects: interactions between unaffected and affected sides. Brain 134:721-731 
54. Lemon RN (2008) Descending pathways in motor control. Annu Rev Neurosci 31:195-218

55. Faist $M$ et al (1999) Impaired modulation of quadriceps tendon jerk reflex during spastic gait: differences between spinal and cerebral lesions. Brain 122 (Pt 3):567-579

56. Thilmann AF et al (1990) Pathological stretch reflexes on the "good" side of hemiparetic patients. J Neurol Neurosurg Psychiatry 53:208-214

57. Dietz V et al (2007) Spastic movement disorder: impaired reflex function and altered muscle mechanics. Lancet Neurol 6:725-733

58. Dyer JO et al (2009) Transmission in heteronymous spinal pathways is modified after stroke and related to motor incoordination. PloS One 4:e4123

59. Forrester LW et al (2008) Exercise-mediated locomotor recovery and lower-limb neuroplasticity after stroke. J Rehabil Res Dev 45:205-220
60. Luft AR et al (2008) Treadmill exercise activates subcortical neural networks and improves walking after stroke: a randomized controlled trial. Stroke 39:3341-3350

61. Werner $C$ et al (2002) Treadmill training with partial body weight support and an electromechanical gait trainer for restoration of gait in subacute stroke patients: a randomized crossover study. Stroke 33:2895-2901

62. Hornby TG et al (2008) Enhanced gait-related improvements after therapist- versus robotic-assisted locomotor training in subjects with chronic stroke: a randomized controlled study. Stroke 39:1786-1792

63. Lamontagne A et al (2004) Faster is better: implications for speed-intensive gait training after stroke. Stroke 35:2543-2548

64. Plummer P et al (2007) Effects of stroke severity and training duration on locomotor recovery after stroke: a pilot study. Neurorehabil Neural Repair 21:137-151 